\title{
High efficiency proton beam generation through target thickness control in femtosecond laser-plasma interactions
}

\author{
J. S. Green, ${ }^{1, a)}$ A. P. L. Robinson, ${ }^{1}$ N. Booth,${ }^{1}$ D. C. Carroll, ${ }^{1}$ R. J. Dance,${ }^{2}$ R. J. Gray, ${ }^{3}$ \\ D. A. MacLellan, ${ }^{3}$ P. McKenna, ${ }^{3}$ C. D. Murphy, ${ }^{4}$ D. Rusby, ${ }^{1}$ and L. Wilson ${ }^{1}$ \\ ${ }^{1}$ Central Laser Facility, STFC Rutherford Appleton Laboratory, Chilton, Oxon OX11 0QX, United Kingdom \\ ${ }^{2}$ York Plasma Institute, Department of Physics, University of York, York YO10 5DD, United Kingdom \\ ${ }^{3} S U P A$, Department of Physics, University of Strathclyde, Glasgow G4 ONG, United Kingdom \\ ${ }^{4}$ SUPA, University of Edinburgh, Mayfield Road, Edinburgh EH9 3JZ, United Kingdom
}

(Received 21 March 2014; accepted 12 May 2014; published online 27 May 2014)

\begin{abstract}
Bright proton beams with maximum energies of up to $30 \mathrm{MeV}$ have been observed in an experiment investigating ion sheath acceleration driven by a short pulse ( $<50 \mathrm{fs})$ laser. The scaling of maximum proton energy and total beam energy content at ultra-high intensities of $\sim 10^{21} \mathrm{~W}$ $\mathrm{cm}^{-2}$ was investigated, with the interplay between target thickness and laser pre-pulse found to be a key factor. While the maximum proton energies observed were maximised for $\mu \mathrm{m}$-thick targets, the total proton energy content was seen to peak for thinner, $500 \mathrm{~nm}$, foils. The total proton beam energy reached up to $440 \mathrm{~mJ}$ (a conversion efficiency of $4 \%$ ), marking a significant step forward for many laser-driven ion applications. The experimental results are supported by hydrodynamic and particle-in-cell simulations. (C) 2014 Author(s). All article content, except where otherwise noted, is licensed under a Creative Commons Attribution 3.0 Unported License.
\end{abstract}

[http://dx.doi.org/10.1063/1.4879641]

Over recent years, considerable effort has been expended on developing laser-driven ion sources for applications ranging from ion oncology ${ }^{1}$ to proton radiography. ${ }^{2}$ Promising characteristics such as high peak brightness, low emittance, ${ }^{3}$ and short pulse duration make using high intensity lasers for ion acceleration an attractive prospect compared to many conventional accelerator sources.

The drive towards higher proton energies with quasimonoenergetic spectra has peaked interest in new acceleration modes such as radiation pressure acceleration (RPA). ${ }^{4}$ However, there remains broad interest in developing laserdriven beamlines based on the more established mechanism of target normal sheath acceleration (TNSA). ${ }^{5}$ With less stringent requirements on laser intensity, contrast, and targetry, TNSA remains easier to implement on a range of laser systems, and the characteristic quasi-thermal spectrum is well suited to applications such as isochoric heating ${ }^{6}$ and time-resolved radiography. ${ }^{2}$

Many key experiments in the field of laser-accelerated ion beams have been performed on large scale $\mathrm{Nd}$ :Glass laser systems where high laser pulse energies $(>100 \mathrm{~J})$ coupled with sub-picosecond pulse lengths have produced focused intensities of $\sim 10^{21} \mathrm{~W} \mathrm{~cm}^{-2}$ to accelerate the highest energy protons $(\sim 60 \mathrm{MeV}) .^{7-9}$ However, recent years have seen the commissioning of an increasing number of high intensity Ti:sapphire based lasers which operate at pulse lengths typically around $50 \mathrm{fs}$ but with lower pulse energies $(\sim 0.5-10 \mathrm{~J})$. Such systems have the advantage that they not only occupy a smaller spatial footprint but also operate at significantly higher repetition rates (typically 1 shot per minute here) when compared to similar intensity Nd:Glass lasers ( $\sim 1$ shot per hour). Many conceivable applications of laser-accelerated ion beams will not only require a low cost,

${ }^{a)}$ Electronic mail: james.green@stfc.ac.uk compact system but also require operation at high repetition rates $(>10 \mathrm{~Hz})$. Ti:sapphire lasers are most likely to fulfil these requirements, hence experimental effort to characterise and optimise ion acceleration processes using femtosecond laser sources is a crucial step towards many proof of principle experiments.

Until recently, results with Ti:sapphire systems have been limited to maximum proton energies of $<20 \mathrm{MeV},{ }^{10,11}$ with thin $(<1 \mu \mathrm{m})$ targets typically used to maximise these energies for a given laser intensity. However, in the last two years, promising gains have been made using ultra-short pulses with Ogura et al. ${ }^{12}$ and Kim et al. ${ }^{13}$ reporting maximum proton energies of $40 \mathrm{MeV}$ and $45 \mathrm{MeV}$, respectively.

In this paper, we report on an experimental investigation into ion acceleration on a Ti:sapphire-based laser which can produce a focused intensity of $\sim 10^{21} \mathrm{~W} \mathrm{~cm}^{-2}$. This is comparable to the highest intensity Nd:Glass systems but in a relatively compact area and operating at a significantly higher repetition rate. The interaction of this laser with aluminium target foils, ranging in thickness from $100 \mathrm{~nm}$ to $50 \mu \mathrm{m}$, generated proton beams which were then characterised. The variation of the maximum proton energy as well as the total beam energy as a function of target thickness will be shown. We demonstrate that a Ti:sapphire based laser system can not only be used to produce proton energies in excess of $30 \mathrm{MeV}$ through TNSA but also produces high average doses, marking an important breakthrough for high repetition rate studies.

The experiment was performed using the Astra Gemini laser ${ }^{14}$ at the Central Laser Facility. Astra Gemini is a dualbeam Ti:sapphire $800 \mathrm{~nm}$ laser that delivers $\sim 10 \mathrm{~J}$ of energy on target per beam with a pulse duration of $45 \mathrm{fs}$. One arm of Astra Gemini was focused onto target at $30^{\circ}$ incidence with a p-polarised orientation, using an $\mathrm{f} / 2$ parabola resulting in a Full Width Half Maximum (FWHM) spot size of $2.5 \mu \mathrm{m}$. 


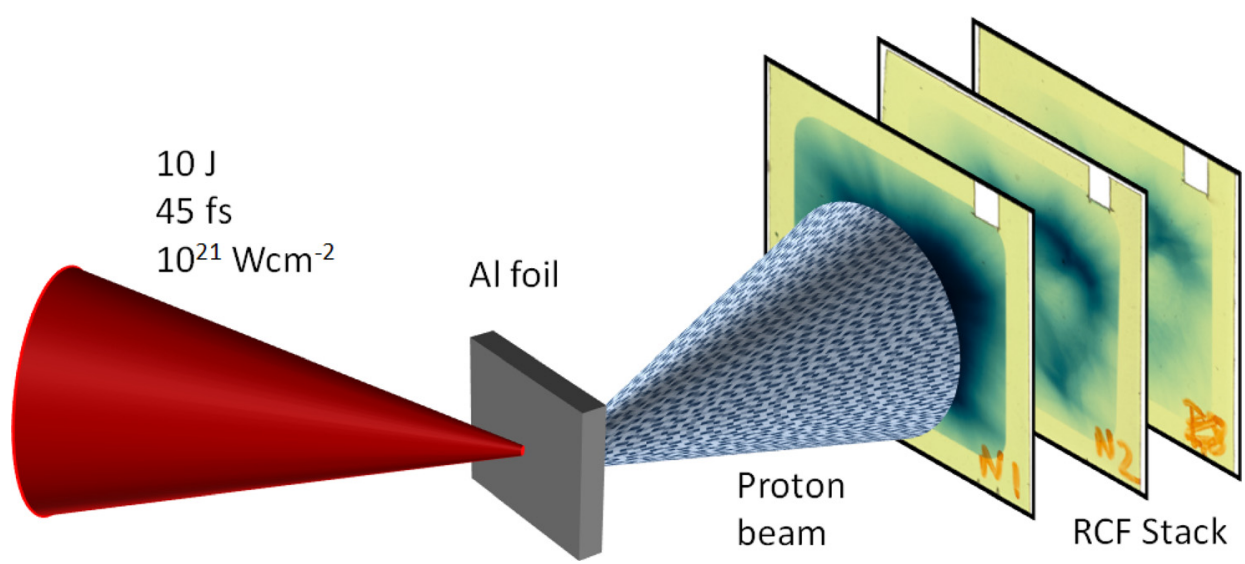

FIG. 1. Overview of experimental setup showing Astra-Gemini interaction beam and RCF stack. The RCF stack was positioned $45 \mathrm{~mm}$ from the rear surface of the target in order to capture the full proton beam.

Approximately $30 \%$ of the incident laser energy was contained within the central focal spot, resulting in a peak intensity of $\sim 10^{21} \mathrm{~W} \mathrm{~cm}^{-2}$. The level of amplified spontaneous emission (ASE) of the laser was characterised using a third-order scanning autocorrelator. The contrast ratio (the ratio between ASE intensity and the peak of the main interaction pulse) of the ASE pedestal was measured to be $\sim 10^{10}$ up to $20 \mathrm{ps}$ before the main pulse, yielding a nanosecondASE intensity of $\sim 10^{11} \mathrm{~W} \mathrm{~cm}{ }^{-2}$. Prior to $1 \mathrm{~ns}$ before the main pulse Pockels cells prevented any additional ASE reaching the target.

Aluminium target foils were mounted into $5 \times 5$ array pucks, with each target being $1 \mathrm{~mm}$ in diameter and thickness varying from $100 \mathrm{~nm}$ to $50 \mu \mathrm{m}$. A high resolution (f/2) microscope objective lens was driven in behind the targets before each shot in order to position each target foil in the focal plane of the laser. The proton beam was characterised by a $2.5 \mathrm{~cm}$ square radiochromic film (RCF) stack that was placed $45 \mathrm{~mm}$ behind the target, normal to the rear surface (Figure 1). Typical full angle beam divergence was $<30^{\circ}$ over all target thicknesses, meaning that the whole proton beam was recorded for most shots. Each stack consisted of a number of dosimetry films that were sandwiched together in order to record the spatial profile and dose over a range of energies. Gafchromic HD-V2 was typically used for the first 5 layers of the stack in order to record the highest doses, with Gafchromic EBT2 used to record the highest energies where typically the dose is significantly lower. For each design of RCF stack, the ion transport code SRIM $^{15}$ was used to model the range of proton energies stopped in each layer. Since ions exhibit a Bragg peak stopping profile, whereby the peak energy loss occurs at the end of their range, each RCF layer can be considered to be $2 \mathrm{D}$ dose profile sampled at a discrete energy. A $13 \mu \mathrm{m}$ thick layer of Aluminium foil was placed at the front of each stack in order to shield the stack from debris and minimise any dose contribution from any heavy ions. The presence of this foil limited the minimum detectable proton energy to $0.9 \mathrm{MeV}$.

In order to characterise the process of TNSA at the ultra-high intensities available on the Astra Gemini system, a range of Aluminium target foils were irradiated under optimal focus conditions. The maximum proton energy detected as a function of target thickness was measured over a series of shots (Figure 2), where laser pulse energy fluctuated by no more than $10 \%$. The maximum energy was determined by identifying the last layer of RCF that had a detectable proton dose. The error bars in Figure 2 were largely determined by the variation in maximum energy over multiple repeat data shots for each target thickness, although the uncertainty from the discrete energy windows in the RCF design is also included. The variation in peak energy for each target thickness is likely due to a combination of factors, namely, accuracy in target positioning, changes in laser pulse energy, and stochastic variations in the pre-pulse profile. The highest detectable proton energy can be seen to increase rapidly as the target thickness is decreased from $50 \mu \mathrm{m}$ to $6 \mu \mathrm{m}$, peaking for some shots at over $30 \mathrm{MeV}$. However, as the target thickness is reduced further from $2 \mu \mathrm{m}$ to $100 \mathrm{~nm}$, the maximum proton energy rapidly tails off to an average of just $10 \mathrm{MeV}$.

Experimental results from several laser systems illustrate that for fixed laser parameters, there will be an optimum target thickness for ion acceleration, at which the fast electron density that seeds the accelerating field at the target rear surface is maximised and the initial plasma-vacuum interface is still close to step-like. ${ }^{16-18}$ The trend from Figure 2 exhibits a similar profile to these results, suggesting that while a target thickness in the range of $2-6 \mu \mathrm{m}$ is optimal in this case, acceleration in thinner targets may be inhibited by the presence of pre-pulse induced plasma formation on the target rear surface. ${ }^{19,20}$

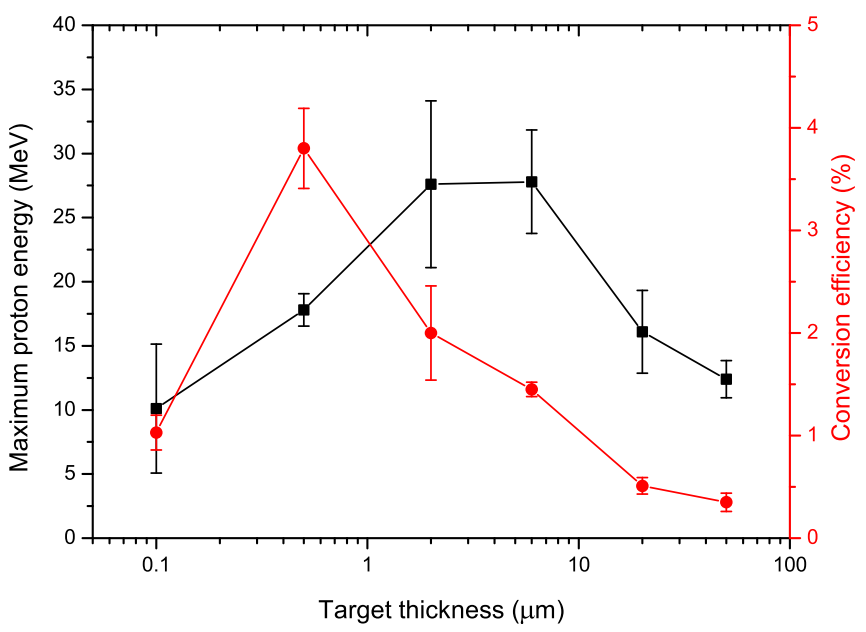

FIG. 2. Maximum detected proton energy (squares) and conversion efficiency (circles, for $\mathrm{E}_{p}>0.9 \mathrm{MeV}$ ) as a function of target thickness for $\mathrm{Al}$ foils. Data plotted are averages taken over a number of shots for each target thickness. 
To investigate this further, the total proton dose (extracted from each RCF stack) for each target thickness was calculated (see Figure 2). The dose deposited in each RCF layer was calculated by converting the optical density map for each film into a dose distribution using a calibrated set of films, which were irradiated with a known dose at a cyclotron facility. The total dose in each layer, together with proton stopping curves calculated using SRIM, was then used to extract the proton spectrum for each shot. Finally, the conversion efficiency was calculated by dividing the total proton beam energy deposited in the RCF stack by the laser energy on target.

As the target thickness is decreased from $50 \mu \mathrm{m}$ to $500 \mathrm{~nm}$, a clear increase in conversion efficiency is observed, peaking at just over $4 \%$ for one shot, corresponding to an energy content of $\sim 440 \mathrm{~mJ}$ inside the proton beam. This is over twice that of the average value for the $6 \mu \mathrm{m}$ foil thickness $(1.5 \%, \sim 160 \mathrm{~mJ})$, for which the maximum proton energy is optimised. Examination of the extracted spectra reveals that this peak in conversion efficiency is dominated by a much greater number of lower energy $(<15 \mathrm{MeV})$ protons (see Figure 3). $440 \mathrm{~mJ}$ is the highest proton beam energy content reported from a Ti:sapphire based laser system. Similar conversion efficiencies have been seen experimentally, for example, $3 \%$ by Nishiuchi et al. ${ }^{21}$ but with a significantly lower laser energy. The results detailed here demonstrate that by scaling up the laser pulse energy to $10 \mathrm{~J}$, an even greater efficiency is obtained. And while the peak proton energy is reduced in this optimised case, the energy range below $15 \mathrm{MeV}$ is highly relevant for current investigations into warm dense matter ${ }^{22}$ or biological damage studies. $^{23,24}$

In order to better understand the regime of TNSA with the laser and target parameters being considered here, the 1D radiation-hydrodynamic code HELIOS ${ }^{25}$ was first used to model the possible disruptive effects of ASE on the thinnest target foils. An ASE intensity of $10^{11} \mathrm{~W} \mathrm{~cm}^{-2}$, as measured from the autocorrelation scan, was used to irradiate $100 \mathrm{~nm}$ and $500 \mathrm{~nm}$ target foils along with a thicker $6 \mu \mathrm{m}$ foil for reference. The target mass densities were plotted after $1 \mathrm{~ns}$ of

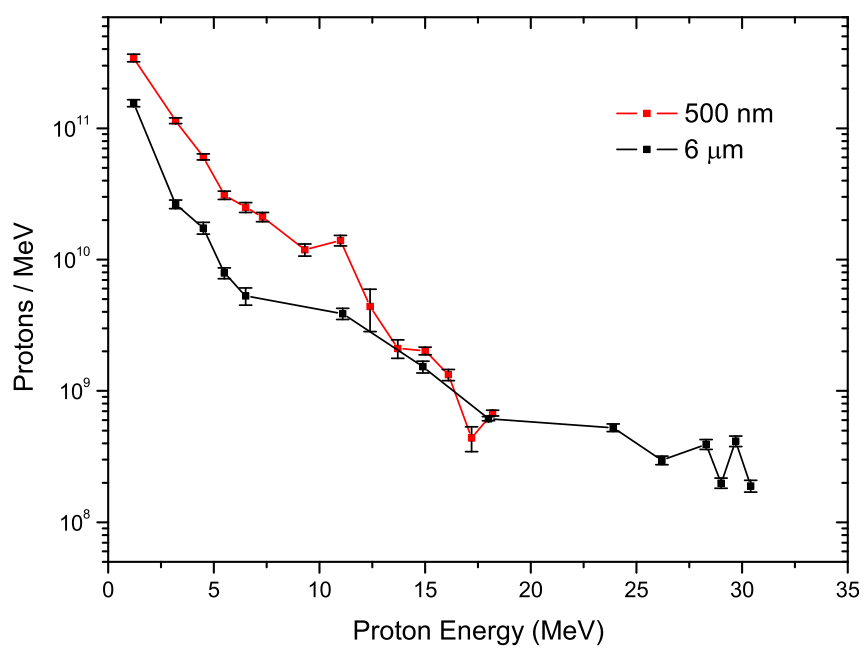

FIG. 3. Experimentally obtained proton spectra, extracted from RCF stacks for $500 \mathrm{~nm}$ and $6 \mu \mathrm{m} \mathrm{Al}$ foil targets. simulation time (see Figure 4), representing the conditions present at the time of the interaction of the main pulse.

For the thicker $(6 \mu \mathrm{m})$ foil, target pre-plasma expansion is evident at the target front surface, but the bulk of the target remains at solid density $\left(2.7 \mathrm{~g} \mathrm{~cm}^{-3}\right)$, leaving a steep density transition at the rear surface (as required for optimal sheath acceleration). As the target thickness is reduced, a similar front surface density ramp remains, but the profile at the rear surface is significantly disrupted for both the $500 \mathrm{~nm}$ and $100 \mathrm{~nm}$ cases, indicating non-ideal acceleration conditions.

Three models of the target at the time of the main pulse arriving were produced based on the aforementioned hydrodynamic simulations (Figure 4), and the interactions of these model targets with the main pulse were simulated using the OSIRIS 2D3V particle-in-cell (PIC) code. ${ }^{26}$ An $8000 \times 8000$ grid was used for a simulation box of $32 \times 32 \mu \mathrm{m}$. All targets were centred in the $y$-direction and had a width of $20 \mu \mathrm{m}$ with a top-hat profile in the $y$-direction. The $x$-profile and composition of the targets were determined from the output of the HELIOS simulations. In all three models, the linearly polarized laser pulse was incident at $30^{\circ}$ to target normal and centred in the $y$-direction. The laser pulse had a normalised vector potential, $a_{0}$, of 17 , a triangular temporal profile with a pulse length of $50 \mathrm{fs}$, and a Gaussian transverse profile with a half-width of $1.5 \mu \mathrm{m}$. Each species was represented by 16 macroparticles per cell. Simulations were run up to 250 fs. The initial electron temperature in the target was set to $4 \mathrm{keV}$.

The integrated proton spectra obtained from all three runs are shown in Figure 5. Looking first at the maximum proton energy, it is clear that the cut-off energy follows a similar trend to that seen experimentally, with the peak proton energy resulting from the thicker, $6 \mu \mathrm{m}$ target. Taking a cut-off point of around $10^{5}$ protons $/ \mathrm{MeV}$ (corresponding to where the $500 \mathrm{~nm}$ spectrum tails off rapidly), the maximum proton energy is recorded as 33,28 , and $18 \mathrm{MeV}$ for the $6 \mu \mathrm{m}, 500 \mathrm{~nm}$, and $100 \mathrm{~nm}$ foils, respectively (see Figure 6). While higher than measured experimentally, the trend is consistent. The presence of an extended pre-plasma at the target front surface, while common to each target thickness, has the

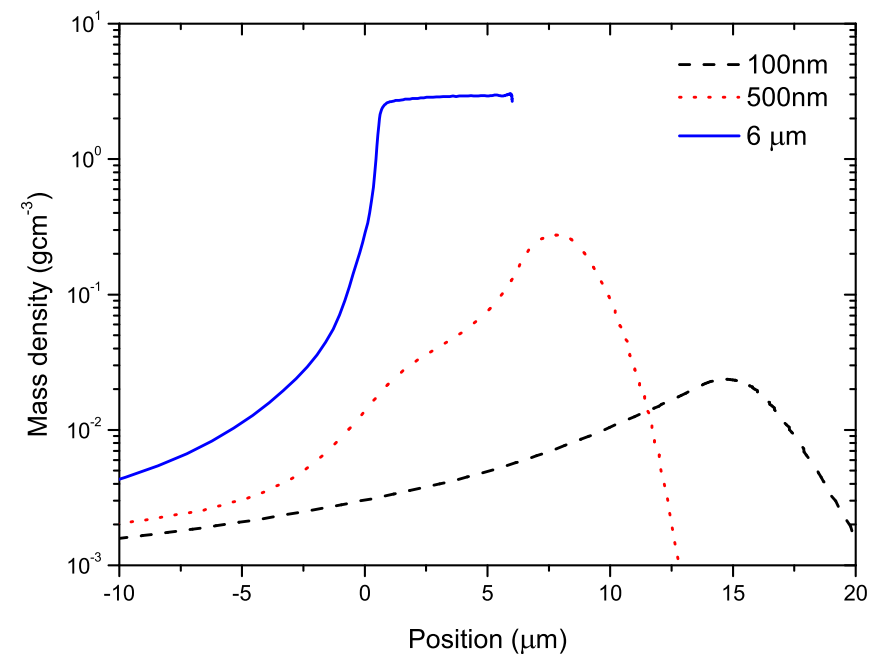

FIG. 4. Simulated target density profiles at the point that the main laser pulse interacts with each target (laser is incident from the left hand side), modelling using HELIOS. The initial target front surface position is $0 \mu \mathrm{m}$. 


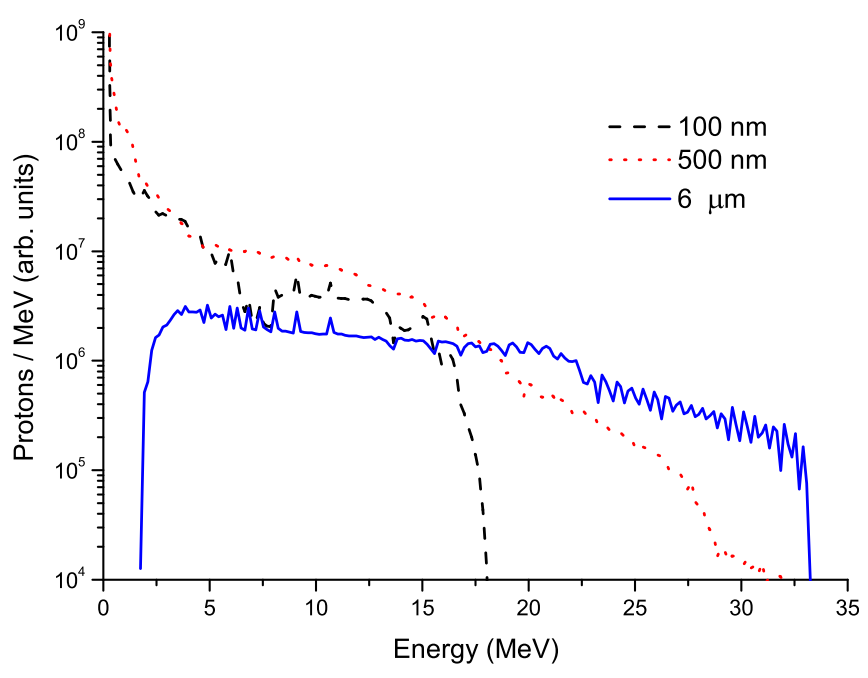

FIG. 5. Simulated proton spectra (spatially and temporally integrated over the whole beam) obtained from PIC simulations for three target thicknesses. For each target thickness, the initial density profile was determined from the output from HELIOS (Figure 4). Note: Proton numbers are given in arbitrary units.

effect of efficiently coupling the laser energy into the fast electron population. ${ }^{27}$ To illustrate this, an additional simulation was run with a step-like density gradient at the target front surface for the $6 \mu \mathrm{m}$ thickness. For this case, the peak proton energy was seen to fall by $\sim 25 \%$.

Looking at the spectra in Figure 5 in more detail, it can be seen that the experimentally observed boost in lower energy $(<15 \mathrm{MeV})$ proton number for $500 \mathrm{~nm}$ is also reproduced. Taking the integrated proton number for each target thickness, the $500 \mathrm{~nm}$ foil produces a total proton beam energy six times greater than for the $6 \mu \mathrm{m}$ case, with this increase dominated by proton energies lower than $15 \mathrm{MeV}$ (see Figure 6). While it is clear that to obtain the very highest proton energies, a step-like rear surface density is still required, these simulations highlight that the presence of a modest pre-formed plasma on the target rear surface can still permit efficient TNSA. For the $500 \mathrm{~nm} \mathrm{Al}$ foil, there exists a balance whereby a large electron number density at the

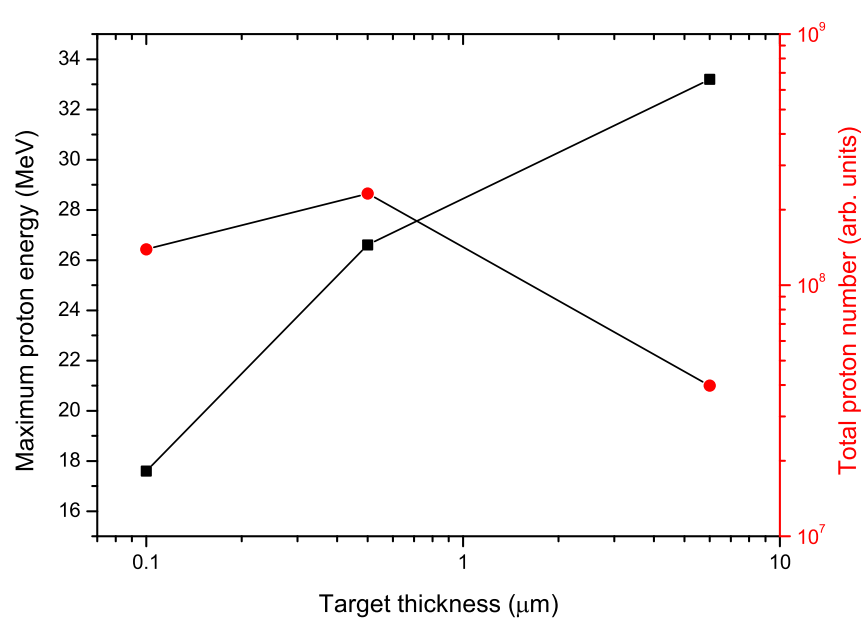

FIG. 6. Maximum proton energies (squares) and integrated proton number (circles), as calculated from PIC simulations. Note: Proton number is sourced from macro-particles in the simulation and so given in arbitrary units. target rear surface (due to the reduced thickness) can drive significant proton acceleration without the need for a steplike interface. In addition, the simulations show that the initial density gradient on the rear surface leads to a piston-like acceleration, ${ }^{28}$ whereby protons accelerated in the highdensity region (close to the target) catch up with those accelerated from the low-density region early in the acceleration process. This piston-like action leads to bunching of the proton population, boosting the lowest energy proton flux significantly. As the target gets thinner still $(100 \mathrm{~nm})$ and further decompression occurs this is no longer the case, with both peak proton energies and total number falling.

We have investigated the production of high energy proton beams from an ultra-intense, Ti:sapphire based laser in the TNSA regime. Using RCF stacks, the maximum proton energies and beam energy content were measured over the whole beam profile. Under the laser contrast conditions tested during the experiment, it was found that the maximum proton energy peaked for target thicknesses in the range of 2-6 $\mu \mathrm{m}$. However, the total energy contained in the recorded proton beam was found to be significantly higher for the $500 \mathrm{~nm}$ foil, peaking at $\sim 440 \mathrm{~mJ}$ for one shot, corresponding to a laser-proton conversion efficiency of around $4 \%$.

Simulations together with experimental proton spectra reveal that this boost in proton number is dominated by lower energy $(<15 \mathrm{MeV})$ particles. Although ASE-induced plasma formation on the target rear surface limits the maximum energy for such thicknesses, highly efficient proton beam production is still achieved. The high proton numbers demonstrated here using a robust acceleration mechanism are highly promising for a range of applications that require bright proton beams under high repetition-rate conditions. We believe these results to be a clear marker of the future capability of laser-driven ion applications with lasers like Astra Gemini, where technology development for future scaling to $10 \mathrm{~Hz} / 100 \mathrm{~J}$ operation within the next few years is rapidly progressing. ${ }^{29}$

This work was supported by the EPSRC (Grant No. $\mathrm{EP} / \mathrm{J} 003832 / 1)$. The authors would like to thank the staff at the Central Laser Facility for supporting the experimental campaign.

${ }^{1}$ S. Bulanov and V. Khoroshkov, Plasma Phys. Rep. 28, 453 (2002).

${ }^{2}$ M. Borghesi, D. Campbell, A. Schiavi, M. Haines, O. Willi, A. MacKinnon, P. Patel, L. Gizzi, M. Galimberti, R. Clarke et al., Phys. Plasmas 9, 2214 (2002).

${ }^{3}$ T. Cowan, J. Fuchs, H. Ruhl, A. Kemp, P. Audebert, M. Roth, R. Stephens, I. Barton, A. Blazevic, E. Brambrink et al., Phys. Rev. Lett. 92, 204801 (2004).

${ }^{4}$ T. Esirkepov, M. Borghesi, S. Bulanov, G. Mourou, and T. Tajima, Phys. Rev. Lett. 92, 175003 (2004).

${ }^{5}$ S. Wilks, A. Langdon, T. Cowan, M. Roth, M. Singh, S. Hatchett, M. Key, D. Pennington, A. MacKinnon, and R. Snavely, Phys. Plasmas 8, 542 (2001).

${ }^{6}$ P. Patel, A. Mackinnon, M. Key, T. Cowan, M. Foord, M. Allen, D. Price, H. Ruhl, P. Springer, and R. Stephens, Phys. Rev. Lett. 91, 125004 (2003).

${ }^{7}$ S. P. Hatchett, C. G. Brown, T. E. Cowan, E. A. Henry, J. S. Johnson, M. H. Key, J. A. Koch, A. B. Langdon, B. F. Lasinski, R. W. Lee et al., Phys. Plasmas 7, 2076 (2000).

${ }^{8}$ L. Robson, P. Simpson, R. Clarke, K. Ledingham, F. Lindau, O. Lundh, T. McCanny, P. Mora, D. Neely, C.-G. Wahlström et al., Nat. Phys. 3, 58 (2007). 
${ }^{9}$ S. Gaillard, T. Kluge, K. Flippo, M. Bussmann, B. Gall, T. Lockard, M. Geissel, D. Offermann, M. Schollmeier, Y. Sentoku et al., Phys. Plasmas 18, 056710 (2011).

${ }^{10}$ K. Zeil, S. Kraft, S. Bock, M. Bussmann, T. Cowan, T. Kluge, J. Metzkes, T. Richter, R. Sauerbrey, and U. Schramm, New J. Phys. 12, 045015 (2010).

${ }^{11}$ M. Schnürer, A. Andreev, S. Steinke, T. Sokollik, T. Paasch-Colberg, P. Nickles, A. Henig, D. Jung, D. Kiefer, R. Hörlein et al., Laser Part. Beams 29, 437 (2011).

${ }^{12}$ K. Ogura, M. Nishiuchi, A. S. Pirozhkov, T. Tanimoto, A. Sagisaka, T. Z. Esirkepov, M. Kando, T. Shizuma, T. Hayakawa, H. Kiriyama et al., Opt. Lett. 37, 2868 (2012).

${ }^{13}$ I. J. Kim, K. H. Pae, C. M. Kim, H. T. Kim, J. H. Sung, S. K. Lee, T. J. Yu, I. W. Choi, C.-L. Lee, K. H. Nam et al., Phys. Rev. Lett. 111, 165003 (2013).

${ }^{14}$ C. Hooker, J. Collier, O. Chekhlov, R. Clarke, E. Divall, K. Ertel, B. Fell, P. Foster, S. Hancock, A. Langley et al., J. Phys. IV 133, 673-677 (2006).

${ }^{15}$ J. F. Ziegler, M. D. Ziegler, and J. P. Biersack, Nucl. Instrum. Methods Phys. Res., Sect. B 268, 1818 (2010).

${ }^{16}$ D. Neely, P. Foster, A. Robinson, F. Lindau, O. Lundh, A. Persson, C.-G. Wahlstrom, and P. McKenna, Appl. Phys. Lett. 89, 021502 (2006).

${ }^{17}$ M. Kaluza, J. Schreiber, M. Santala, G. Tsakiris, K. Eidmann, J. Meyer-ter Vehn, and K. Witte, Phys. Rev. Lett. 93, 045003 (2004).

${ }^{18}$ T. Ceccotti, A. Lévy, H. Popescu, F. Réau, P. d'Oliveira, P. Monot, J. Geindre, E. Lefebvre, and P. Martin, Phys. Rev. Lett. 99, 185002 (2007).

${ }^{19}$ F. Lindau, O. Lundh, A. Persson, P. McKenna, K. Osvay, D. Batani, and C.-G. Wahlström, Phys. Rev. Lett. 95, 175002 (2005).
${ }^{20}$ A. Andreev, S. Steinke, T. Sokollik, M. Schnürer, S. Ter Avetsiyan, K. Y. Platonov, and P. Nickles, Phys. Plasmas 16, 013103 (2009).

${ }^{21}$ M. Nishiuchi, H. Daido, A. Yogo, S. Orimo, K. Ogura, J. Ma, A. Sagisaka, M. Mori, A. Pirozhkov, H. Kiriyama et al., Phys. Plasmas 15, 053104 (2008).

${ }^{22}$ T. White, J. Vorberger, C. Brown, B. Crowley, P. Davis, S. Glenzer, J. Harris, D. Hochhaus, S. Le Pape, T. Ma et al., Sci. Rep. 2, 889 (2012).

${ }^{23}$ S. Kraft, C. Richter, K. Zeil, M. Baumann, E. Beyreuther, S. Bock, M. Bussmann, T. Cowan, Y. Dammene, W. Enghardt et al., New J. Phys. 12, 085003 (2010).

${ }^{24}$ K. Zeil, M. Baumann, E. Beyreuther, T. Burris-Mog, T. Cowan, W. Enghardt, L. Karsch, S. Kraft, L. Laschinsky, J. Metzkes et al., Appl. Phys. B 110, 437 (2013).

${ }^{25}$ J. MacFarlane, I. Golovkin, and P. Woodruff, J. Quant. Spectrosc. Radiat. Transfer 99, 381 (2006)

${ }^{26}$ R. A. Fonseca, L. O. Silva, F. Tsung, V. K. Decyk, W. Lu, C. Ren, W. B. Mori, S. Deng, S. Lee, T. Katsouleas et al., Computational Science-ICCS 2002 (Springer, 2002), pp. 342-351.

${ }^{27}$ D. Batani, R. Jafer, M. Veltcheva, R. Dezulian, O. Lundh, F. Lindau, A. Persson, K. Osvay, C. Wahlström, D. Carroll et al., New J. Phys. 12, 045018 (2010).

${ }^{28}$ T. Grismayer and P. Mora, Phys. Plasmas 13, 032103 (2006).

${ }^{29}$ K. Ertel, S. Banerjee, P. D. Mason, P. J. Phillips, R. J. S. Greenhalgh, C. Hernandez-Gomez, and J. L. Collier, SPIE Optics + Optoelectronics (International Society for Optics and Photonics, 2013), pp. 87801W-87801W. 